\title{
A Source Problem for the Helmholtz Equation via a Dirichlet-to- Neumann Map
}

\author{
Kuo-Ming Lee $\mathbb{D}$ \\ Department of Mathematics, National Cheng Kung University, Taiwan \\ Correspondence should be addressed to Kuo-Ming Lee; kmlee@mail.ncku.edu.tw
}

Received 29 June 2021; Accepted 14 August 2021; Published 23 August 2021

Academic Editor: Yufeng Xu

Copyright (c) 2021 Kuo-Ming Lee. This is an open access article distributed under the Creative Commons Attribution License, which permits unrestricted use, distribution, and reproduction in any medium, provided the original work is properly cited.

In this paper, we consider a source problem for a time harmonic acoustic wave in two-dimensional space. Based on the boundary integral equation method, a Dirichlet-to-Neumann map in terms of boundary integral operators on the boundary of the source is constructed to transform this problem into two boundary value problems for the Helmholtz equation.

\section{Introduction}

The realization of many physical phenomena mathematically leads to the resolution of the Helmholtz equation in a bounded or an unbounded domain with suitable conditions, for example, radiation or scattering of waves $[1,2]$ and swirling flow in the fluid mechanics [3].

Using the boundary integral equation (BIE) approach, the above problems can be transformed into a Fredholm integral equation of the second kind or a system of the same kind. This is beneficial both from a theoretical and a numerical point of view. Theoretically, it gives an elegant analysis of the unique solvability and the stability of the solution based on the power of functional analysis. Numerically, it reduces the computational costs by decreasing the dimension.

Now, consider the scattering problem of a time harmonic acoustic wave with a source. Mathematical formulation for this kind of problem is the resolution of an inhomogeneous Helmholtz equation. This can also be used to model a cavity within homogeneous media. Unlike in the case of a homogeneous equation where only boundary integrals appear [4-6], using boundary integral equation methods in dealing with a source problem usually leads to some boundary integrals and a domain integral [7-9]. Thus, one of the most significant advantages in the BIE structure is demolished in the sense that the dimension is not reduced because of the appearance of the domain integral. It was shown in [10] that the domain integral can be spared if the source term is harmonic. A numerical example for a constant source was also given there.

Starting from this point, we consider the scattering problem with a source from a different perspective. Inspired by [11] where a Dirichlet-to-Neumann (DtN) map is constructed for a transmission scattering problem, we would like to build a DtN map for the source problem. The central idea in [11] is to use the BIE method to construct a DtN map which in turn transforms the transmission scattering problem into a pure boundary value problem for the Helmholtz equation by encoding the information of the interior domain in this map. Sharing this viewpoint, the source problem can be turned into some boundary value problems by a suitable DtN map which contains the source information.

The plan of the paper is as follows. In Section 2, we introduce a source problem and solve it in the usual way via the BIE method. In Section 3, we construct a DtN map as a boundary integral operator. In Section 4, the original source problem is converted into two boundary value problems in different domains for the Helmholtz equation via the DtN map. It is then followed by some numerical examples in Section 5. Some conclusions are given in the final section.

\section{A Source Problem}

Let $D_{s} \subsetneq D_{m} \subsetneq \mathbb{R}^{2}$ be two simply connected open sets with $C^{2}$ boundaries $\Gamma_{s}, \Gamma_{m}$, respectively. Set $D=D_{m} \backslash \bar{D}_{s}$. Besides, we 
assume that the wave number $k \in \mathbb{C}$ is not an interior Dirichlet eigenvalue for the Helmholtz equation in $D_{m}$ throughout this paper.

Let us consider the following source problem.

Problem 1. (source problem for $D_{m}$ ). Given $C^{2}$ domains $D_{s} \subsetneq D_{m} \subsetneq \mathbb{R}^{2}$ and continuous functions $s \neq 0 \in C\left(D_{s}\right), f \in C$ $\left(\Gamma_{m}\right)$, find a solution $u \in C^{2}\left(D \cup D_{s}\right) \cap C^{1}(\bar{D})$ that satisfies the following conditions:

$$
\begin{aligned}
\Delta u+k^{2} u & =\sin D_{s} \\
\Delta u+k^{2} u & =0 \operatorname{in} D \\
u & =f \text { on } \Gamma_{m}
\end{aligned}
$$

For this problem, we propose two different solution methods. The first of which is to split the problem into two parts: one inhomogeneous part without boundary condition and one homogeneous part with boundary condition on the measurement curve $\Gamma_{m}$.

To this aim, we first assume that a particular (free space) solution $u_{p}$ for (1) is given. Then, by the superposition principle for linear equations, a solution for the source problem can be given by

$$
u=\left\{\begin{array}{l}
v \operatorname{in} D, \\
v+u_{p} \operatorname{in} D_{s},
\end{array}\right.
$$

with a solution $v$ to the Helmholtz equation in $D_{m}$. Based on the boundary integral equation method, a solution ansatz for $v$ can be given by the potential

$v(x)=\int_{\Gamma_{s}}\left(\frac{\partial \Phi(x, y)}{\partial v(y)} u_{p}(y)-\Phi(x, y) \frac{\partial u_{p}(y)}{\partial v(y)} d s(y)+\int_{\Gamma_{m}} \frac{\partial \Phi(x, y)}{\partial v(y)} \varphi(y) d s(y)\right)$,

for $x \in D_{s} \cup D$ where

$$
\Phi(x, y):=\frac{i}{4} H_{0}^{(1)}(k|x-y|), \quad x \neq y,
$$

denotes the fundamental solution of the Helmholtz equation in $\mathbb{R}^{2}$ in terms of the Hankel function $H_{0}^{(1)}$ of the first kind and order zero. We note also here that the function $v$ denotes the unit normal directs into the exterior of the domain considered.

For brevity and further discussions, we denote the first part of $v$ by $V_{s}\left(u_{p}\right)$. It can be seen as Green's representation formula for Helmholtz equation in the exterior (to $D_{s}$ ); see $[1,12]$. Since the original problem has one boundary condition on $\Gamma_{m}$, it is natural to add a potential on $\Gamma_{m}$ in the spirit of the boundary integral equation method. The choice of a double layer potential on $\Gamma_{m}$ corresponds to the solution to an interior Dirichlet problem. It is noted at this place that different potentials can be used here, even potentials on some other curves. We will demonstrate this later in the numerical experiments.

With the solution ansatz (5), using the jump relations of double layer potential on $\Gamma_{m}$, it can be easily verified that $u$ is the solution of the source problem if $\varphi$ is the solution of the following boundary integral equation:

$$
V_{s}\left(u_{p}\right)(x)-\frac{1}{2} \varphi(x)+\int_{\Gamma_{m}} \frac{\partial \Phi(x, y)}{\partial v(y)} \varphi(y) d s(y)=f(x), \quad x \in \Gamma_{m} .
$$

The assumption that $k$ is not an interior Dirichlet eigenvalue assures the injectivity of this equation. It follows from the Riesz theory $([1,12])$ the unique solvability of this equation. With this solution $\varphi$, the function $v$ defined by (5) is the solution in $D$ and the function $u$ defined by (4) is the solution to the source problem.

The second method we will propose is somewhat different in the sense that the source problem is transformed into a boundary value problem with boundary $\Gamma_{s} \cup \Gamma_{m}$ for the domain $D$ and a boundary value problem for the domain $D_{s}$ with boundary $\Gamma_{s}$. Since we only have one measurement on $\Gamma_{m}$ at the outset, we still need another boundary condition on $\Gamma_{s}$. To this aim, we will need some tool which is to be described in the next section in full details.

\section{A Dirichlet-to-Neumann Map}

As mentioned, to treat the source problem as boundary value problems, we have to find one more extra boundary condition. Since there is already given a condition on $\Gamma_{m}$, it seems natural to win another condition on the other boundary, i.e., $\Gamma_{s}$.

The idea is to pass the source information in $D_{s}$ to its boundary $\Gamma_{s}$ to fulfill our need. Motivated by [11], where a transmission scattering problem is considered, and by [13] for a Laplace equation, we will introduce a Dirichlet-toNeumann map as a boundary integral operator defined on $\Gamma_{s}$ to take over this task.

To this end, we first consider an interior Dirichlet problem for the Helmholtz equation in $D_{s}$ with boundary $\Gamma_{s}$. Adopting the boundary integral equation method, we start from a solution ansatz for the solution

$u_{s}(x):=a \int_{\Gamma_{s}} \Phi(x, y) \varphi(y) d s(y)+b \int_{\Gamma_{s}} \frac{\partial \Phi(x, y)}{\partial v(y)} \varphi(y) d s(y), \quad a, b \in \mathbb{C}$.

In what follows, we take $a=0, b=1$. We note that the same idea works for all choices of $a, b$ (except $a=0=b$ ), only with different operators and with some additional assumptions on the domain, if necessary. From the assumption that $k$ is not an interior Dirichlet eigenvalue and the solution theory for this problem, we have the unique solvability for this interior Dirichlet problem with the solution $u_{s}$ given by (8) which density is the solution to the boundary integral equation 


$$
2 \int_{\Gamma_{s}} \frac{\partial \Phi(x, y)}{\partial v(y)} \varphi(y) d s(y)-\varphi(x)=2 u_{s}(x), \quad x \in \Gamma_{s} .
$$

For a concise description, we define boundary integral operators $S_{\alpha, \beta}, K_{\alpha, \beta}, K_{\alpha, \beta}{ }^{\prime}, T_{\alpha, \beta}: \mathcal{N}(\Gamma \alpha) \longrightarrow C\left(\Gamma_{\beta}\right)$ by

$$
\begin{aligned}
& S_{\alpha, \beta} \varphi(x):=2 \int_{\Gamma_{\alpha}} \Phi(x, y) \varphi(y) d s(y), \\
& K_{\alpha, \beta}{ }^{\prime} \varphi(x):=2 \int_{\Gamma_{\alpha}} \frac{\partial \Phi(x, y)}{\partial v(y)} \varphi(y) d s(y), \\
& K_{\alpha, \beta}^{\prime} \varphi(x):=2 \int_{\Gamma_{\alpha}} \frac{\partial \Phi(x, y)}{\partial v(x)} \varphi(y) d s(y), \\
& T_{\alpha, \beta} \varphi(x):=2 \frac{\partial}{\partial v(x)} \int_{\Gamma_{\alpha}} \frac{\partial \Phi(x, y)}{\partial v(y)} \varphi(y) d s(y),
\end{aligned}
$$

for $x \in \Gamma_{\beta}$ with $\alpha, \beta \in\{s, m\}$. The space $\mathcal{N}\left(\Gamma_{\alpha}\right)$ is defined as the linear space of continuous functions $\varphi$ on $\Gamma_{\alpha}$ with the property that the double layer potential with $\varphi$ as its density has continuous normal derivative from both side if $\alpha=s$ and from the interior side if $\alpha=m$.

In $D_{s}$, applying the normal derivation to (8) and then passing the parameter $x$ to the boundary $\Gamma_{s}$, we obtain

$$
\frac{\partial u_{s}}{\partial v}(x)=\frac{\partial}{\partial v(x)} \int_{\Gamma_{s}} \frac{\partial \Phi(x, y)}{\partial v(y)} \varphi(y) d s(y) \operatorname{on} \Gamma_{s} .
$$

Now, from the solution theory of the interior Dirichlet problem, the operator $\left(K_{s s}-I\right)^{-1}$ is well defined as a bounded operator and the solution to (9) is given by

$$
\varphi=2\left(K_{s s}-I\right)^{-1} u_{s}
$$

From the above two equations, we have

$$
\frac{\partial u_{s}}{\partial v}=\frac{1}{2} T_{s s} \varphi=T_{s s}\left(K_{s s}-I\right)^{-1} u_{s} \text { on } \Gamma_{s}
$$

Thus, without solving the interior problem, we can define a Dirichlet-to-Neumann operator $A: \mathscr{R}\left(\Gamma_{s}\right) \longrightarrow C$ $\left(\Gamma_{s}\right)$ which maps the Dirichlet data of the solution to its Neumann data on $\Gamma_{s}$ by

$$
A:=T_{s s}\left(K_{s s}-I\right)^{-1} \text {, }
$$

where the function space $\mathscr{R}\left(\Gamma_{s}\right)$ consists of functions in $C^{2}\left(D_{s}\right) \cap C\left(\bar{D}_{s}\right)$ with continuous normal derivative from both side. From the above considerations, it follows the following theorem.

Theorem 1. If $k$ is not an interior Dirichlet eigenvalue for the Helmholtz equation in $D_{s}$, the Dirichlet-to-Neumann map $A$ defined by (14) is well defined.

The above theorem says that for a solution to the Helmholtz equation in $D_{s}$, we can define a DtN map on its bound- ary via (14). After this preparation, we can propose an alternative method in the next section.

\section{An Alternative Method Based on a DtN Map}

In this section, we shall provide an alternative method based on the DtN map developed earlier. The idea is to transform the original source problem into two boundary value problems which are to be solved consecutively.

Just as usual, we assume a solution $u$ of the source Problem 1 and look for conditions it must satisfy. Since $u$ solve the Helmholtz equation in $D$, we first consider a boundary value problem in the domain $D$ with the boundary $\Gamma_{m} \cup \Gamma_{s}$ . One condition is given by (3) which is the measurement on $\Gamma_{m}$. However, for this boundary value problem to be unique, we need another condition. Therefore, we seek a boundary condition on the other boundary, i.e., $\Gamma_{s}$.

Given a particular solution $u_{p}$ to (1), it follows that $u-$ $u_{p}$ satisfies the Helmholtz equation in $D_{s}$. Applying the normal derivative to $u-u_{p}$, we have

$$
\frac{\partial\left(u-u_{p}\right)_{-}}{\partial v}=A\left(u-u_{p}\right)_{-} \text {on } \Gamma_{s} .
$$

Making use the fact that both $u$ and $u_{p}$ and their normal derivatives are continuous across $\Gamma_{s}$, we have

$$
\frac{\partial\left(u-u_{p}\right)_{+}}{\partial v}=\frac{\partial\left(u-u_{p}\right)_{-}}{\partial v}=A\left(u-u_{p}\right)_{-}=A\left(u-u_{p}\right)_{+} \text {on } \Gamma_{s} .
$$

Thus, we have a nonlocal Robin-type boundary condition

$$
\frac{\partial u}{\partial v}-A u=\frac{\partial u_{p}}{\partial v}-A u_{p}
$$

on $\Gamma_{s}$ for the domain $D$.

Having 2 boundary conditions at hand, we can now consider the following boundary value problem.

Problem 2. (interior boundary value problem for $D$ ). Find a solution $w \in C^{2}(D) \cap C^{1}(\bar{D})$ that satisfies the following conditions:

$$
\begin{aligned}
\Delta w+k^{2} w & =0 \operatorname{in} D \\
w & =f \operatorname{on} \Gamma_{m} \\
\frac{\partial w}{\partial v}-A w & =\frac{\partial u_{p}}{\partial v}-A u_{p} \text { on } \Gamma_{s}
\end{aligned}
$$

Based on the boundary integral equation method, it is natural to start with a solution ansatz

$$
w(x):=\int_{\Gamma_{s}} \Phi(x, y) \varphi_{s}(y) d s(y)+\int_{\Gamma_{m}} \frac{\partial \Phi(x, y)}{\partial v(y)} \varphi_{m}(y) d s(y), \quad x \in D,
$$


since the boundary for this problem is now $\Gamma_{s} \cup \Gamma_{m}$. We note here that there are many possibilities for the solution ansatz.

Utilizing jump relations for the potentials, (19) and (20) lead to the following system of boundary integral equations:

$$
\begin{gathered}
S_{s m} \varphi_{s}+\left(K_{m m}-I\right) \varphi_{m}=2 f \text { on } \Gamma_{m} \\
\left(K_{s s}^{\prime}+I-A S_{s s}\right) \varphi_{s}+\left(T_{m s}-A K_{m s}\right) \varphi_{m}=2\left(\frac{\partial u_{p}}{\partial v}-A u_{p}\right) \text { on } \Gamma_{s} .
\end{gathered}
$$

It is noted here that the above system can be brought into the following two-by-two matrix form:

$$
(I-B) X=g
$$

with a compact matrix operator $B$.

The following theorem ensures the unique solvability of this system.

Theorem 2. If $k$ is not an interior Dirichlet eigenvalue for the Helmholtz equation in $D_{s} \cup D$, the system (22) and (23) is uniquely solvable.

Proof. We consider the corresponding homogeneous equations to (22) and (23). From the uniqueness of the boundary value Problem 2, we have $w=0$ in $D$. From the continuity, we have $w=0$ on $\Gamma_{s}$. From this and the uniqueness of the interior Dirichlet problem for $D_{s}$, it follows $w=0$ in $D_{s}$. Using the jump relations, we deduce from (23) $\varphi_{s}=0$ and then from (22) $\varphi_{m}=0$. It follows the injectivity of the system (or (24) in matrix form). Then, from the Riesz theory, we have the unique solvability of the system (22) and (23).

After obtaining the solution in $D$, we can now consider the interior Dirichlet problem in $D_{s}$ with boundary value $w-u_{p}$ on $\Gamma_{s}$.

Problem 3. (interior boundary value problem for $D_{s}$ ). Find a solution $v \in C^{2}\left(D_{s}\right) \cap C^{1}\left(\bar{D}_{s}\right)$ that satisfies the following conditions:

$$
\begin{gathered}
\Delta v+k^{2} v=0 \operatorname{in} D_{s} \\
v=w-u_{p} \text { on } \Gamma_{s}
\end{gathered}
$$

Since $k$ is not an interior Dirichlet eigenvalue for the Helmholtz equation in $D_{s}$, this problem has a unique solution $v$ which can be given by the solution ansatz

$$
v(x):=\int_{\Gamma_{s}} \frac{\partial \Phi(x, y)}{\partial v(y)} \psi(y) d s(y), \quad x \in D_{s},
$$

where the density function $\psi$ is the unique solution to the boundary integral equation

$$
\psi(x)-2 \int_{\Gamma_{s}} \frac{\partial \Phi(x, y)}{\partial v(y)} \psi(y) d s(y)=-2\left(w-u_{p}\right)(x), \quad x \in \Gamma_{s} .
$$

After solving Problems 2 and 3, the solution to the original source problem is then given by

$$
u(x)= \begin{cases}w(x), & x \in D \\ v(x)+u_{p}(x), & x \in D_{s}\end{cases}
$$

Summing up, assuming a particular solution $u_{p}$ for the inhomogeneous Helmholtz equation in $D_{s}$, the proposed DtN-based method transforms the source problem into 2 boundary value problems for the Helmholtz equation which are to be solved consecutively by introducing a Dirichlet-to-Neumann map.

The key feature of this method is that, instead of a source problem, we solve two boundary value problems with a common boundary. The information of the source is transferred to its boundary by a DtN map; see also [11] for a penetrable obstacle in the scattering problem. Thus, if one is only interested in the exterior to the source, there is no need to solve Problem 3. This is more interesting from the viewpoint of the inverse problem since the shape and location of the source are unknown.

\section{Numerical Results}

As mentioned earlier, there are many different choices for solution ansatzes that appeared in previous sections. For each of our methods, we choose two different ansatzes for comparison.

For the first method, we choose these two different ansatzes

(B1) $u(x)=V_{s}\left(u_{p}\right)(x)+\int_{\Gamma_{m}} \Phi(x, y) \varphi(y)$,

(B2) $u(x)=V_{s}\left(u_{p}\right)(x)+\int_{\Gamma_{p}} \Phi(x, y) \varphi(y)$, where $\Gamma_{p}$ is an arbitrary (closed) curve other than $\Gamma_{m}, \Gamma_{s}$. For the DtN map-based method, we choose

(D1) $w(x)=\int_{\Gamma_{s}} \Phi(x, y) \varphi_{s}(y) d s(y)+\int_{\Gamma_{m}} \Phi(x, y) \varphi_{m}(y) d s(y)$,

(D2) $w(x)=\int_{\Gamma_{s}}^{s} \Phi(x, y) \varphi_{s}(y) d s(y)+\int_{\Gamma_{m}}^{m}(\partial \Phi(x, y) / \partial v(y))$ $\varphi_{m}(y) d s(y)$.

In each of our examples below, we evaluate the solution at $x_{1}=(1.8,0.3)$ and $x_{2}=(-1.6,0.2)$ and are listed in the upper half and the lower half in each table, respectively. In all the examples, we use solid (black) line to represent the boundary of the source. The measurement is performed on the dashed (red) curve, and the curve $\Gamma_{p}$ is denoted by the dotted (blue) curve. In all examples below, $\Gamma_{m}$ and $\Gamma_{p}$ are taken to be circles centered at the origin with radius $r$ and $1.2 r$, respectively. For $\Gamma_{s}$, we take parametrization of the form

$$
\gamma(t)=\rho(t)(\cos t, \sin t)-V, \quad t \in[0,2 \pi]
$$

with a constant vector $V \in \mathbb{R}^{2}$. 


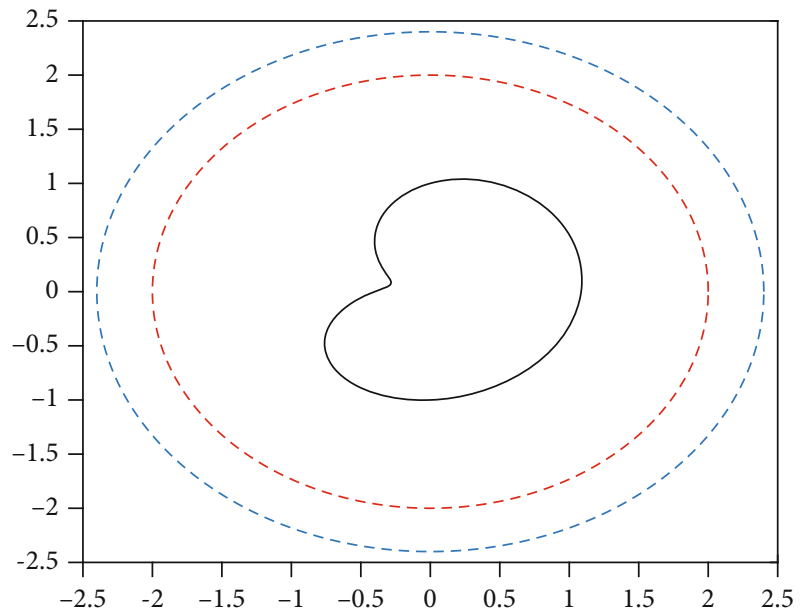

(a) 1: bean, $r=2$

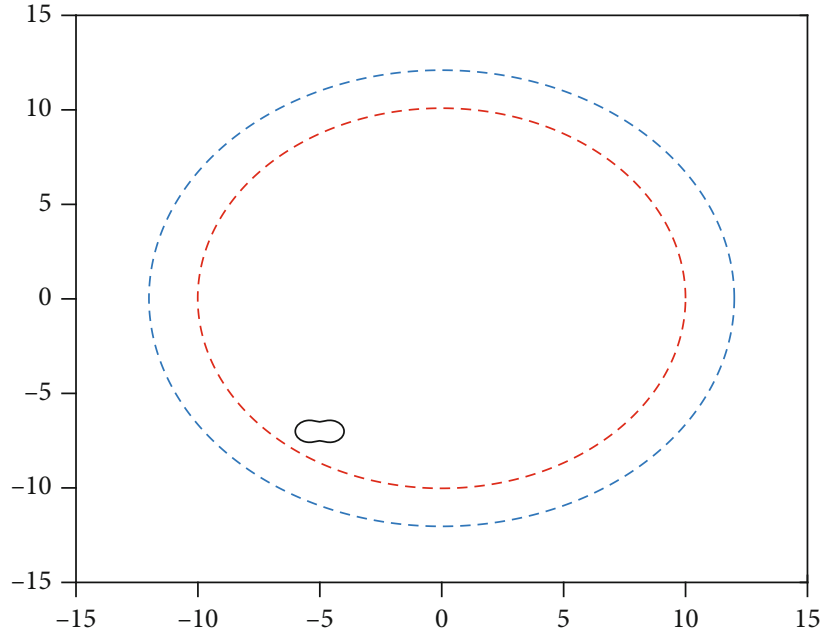

(b) 2: peanut, $r=10$

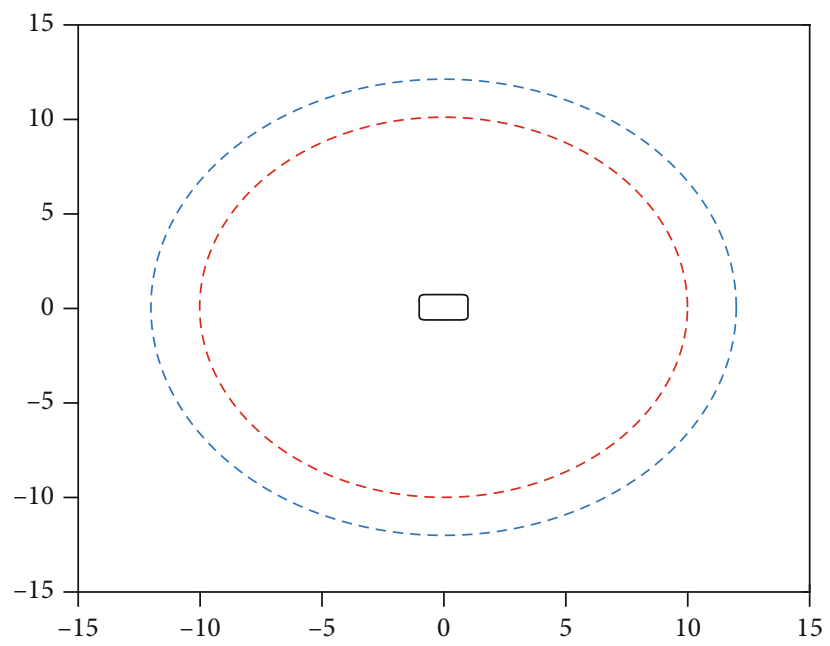

(c) 3: rectangle, $r=10$

Figure 1: Three different geometric configurations.

TABle 1: Bean, $u_{p}=1 / k^{2}$.

\begin{tabular}{lcccc}
\hline$n$ & B1 & B2 & D1 & D2 \\
\hline 16 & -0.3946984858 & -0.3944042796 & -0.3946759466 & -0.4089470621 \\
32 & -0.3943938194 & -0.3944000886 & -0.3943938516 & -0.3936251153 \\
64 & -0.3944000711 & -0.3944000849 & -0.3944000711 & -0.3943971941 \\
128 & -0.3944000849 & -0.3944000849 & -0.3944000849 & -0.3944000849 \\
256 & -0.3944000849 & -0.3944000849 & -0.3944000849 & -0.3944000849 \\
16 & -0.3519607843 & -0.3519740996 & -0.3518944651 & -0.3513983635 \\
32 & -0.3519735225 & -0.3519735244 & -0.3519736158 & -0.3519735938 \\
64 & -0.3519735241 & -0.3519735241 & -0.3519735241 & -0.3519735241 \\
128 & -0.3519735241 & -0.3519735241 & -0.3519735241 & -0.3519735241 \\
256 & -0.3519735241 & -0.3519735241 & -0.3519735241 & -0.3519735241 \\
\hline
\end{tabular}

Example 3. In this example, we consider a bean-shaped source (Figure $1(\mathrm{a}))$ with $\rho(t)=(1+0.9 \cos t+0.1 \sin 2 t) /(1+0.75$ $\cos t), V=(0,0)^{t}$. The aim is to test the influence of the particular solution $u_{p}$. For a source of constant strength $s=1$, we choose $u_{p}=1 / k^{2}$ and $u_{p}(x)=\sin k x+\cos k y+1 / k^{2}$. The results are given in Tables 1 and 2, respectively. From our experiments, we see that the choice of $u_{p}$ has no influence on the outcome. 
TABle 2: Bean, $u_{p}(x)=\sin k x+\cos k y+1 / k^{2}$.

\begin{tabular}{lcccc}
\hline$n$ & B1 & B2 & D1 & D2 \\
\hline 16 & -0.3946981541 & -0.3944039483 & -0.3946762405 & -0.4089473673 \\
32 & -0.3943938194 & -0.3944000886 & -0.3943938516 & -0.3936251153 \\
64 & -0.3944000711 & -0.3944000849 & -0.3944000711 & -0.3943971941 \\
128 & -0.3944000849 & -0.3944000849 & -0.3944000849 & -0.3944000849 \\
256 & -0.3944000849 & -0.3944000849 & -0.3944000849 & -0.3944000849 \\
16 & -0.3519611106 & -0.3519744261 & -0.3518954204 & -0.3513993182 \\
32 & -0.3519735225 & -0.3519735244 & -0.3519736158 & -0.3519735938 \\
64 & -0.3519735241 & -0.3519735241 & -0.3519735241 & -0.3519735241 \\
128 & -0.3519735241 & -0.3519735241 & -0.3519735241 & -0.3519735241 \\
256 & -0.3519735241 & -0.3519735241 & -0.3519735241 & -0.3519735241 \\
\hline
\end{tabular}

Table 3: Peanut, $k=1$.

\begin{tabular}{lcccc}
\hline$n$ & B1 & B2 & D1 & D2 \\
\hline 16 & 0.3096251969 & 0.3068051975 & 0.3096251780 & 0.3096108085 \\
32 & 0.3081918612 & 0.3081887022 & 0.3081918612 & 0.3081918612 \\
64 & 0.3081921023 & 0.3081921023 & 0.3081921023 & 0.3081921023 \\
128 & 0.3081920980 & 0.3081920980 & 0.3081920980 & 0.3081920980 \\
256 & 0.3081920980 & 0.3081920980 & 0.3081920980 & 0.3081920980 \\
16 & -0.2404511673 & -0.2387960788 & -0.2404511597 & -0.2404299904 \\
32 & -0.2395480753 & -0.2395462102 & -0.2395480753 & -0.2395480753 \\
64 & -0.2395509535 & -0.2395509535 & -0.2395509535 & -0.2395509535 \\
128 & -0.2395509515 & -0.2395509515 & -0.2395509515 & -0.2395509515 \\
256 & -0.2395509515 & -0.2395509515 & -0.2395509515 & -0.2395509515 \\
\hline
\end{tabular}

TAble 4: Peanut, $k=10$.

\begin{tabular}{lcccc}
\hline$n$ & B1 & B2 & D1 & D2 \\
\hline 16 & -0.0127033151 & 0.1356744923 & -0.0124653437 & 0.5375881398 \\
32 & 0.2465049437 & 0.0479616266 & 0.2465049437 & 1.3492657328 \\
64 & -0.7568065688 & -2.1566015720 & -0.7568065688 & -2.1769236639 \\
128 & -2.1558807918 & -2.1558787968 & -2.1558807918 & -2.1558787931 \\
256 & -2.1558787968 & -2.1558787968 & -2.1558787968 & -2.1558787968 \\
16 & -0.1058005565 & -0.4641655160 & -0.1061134109 & 1.0485282709 \\
32 & -0.6314665453 & -1.1013082980 & -0.6314665452 & -1.3068114651 \\
64 & 3.5624201062 & 9.1776564238 & 3.5624201062 & 9.2685634343 \\
128 & 9.1775036301 & 9.1775041757 & 9.1775036301 & 9.1775041767 \\
256 & 9.1775041757 & 9.1775041757 & 9.1775041757 & 9.1775041757 \\
\hline
\end{tabular}

Example 4. In this example, we take a peanut-shaped source (Figure 1(b)) with $s=1$ and $\rho(t)=\sqrt{\cos ^{2} t+0.25 \sin ^{2} t}, V=$ $(5,7)^{t}$. We test for different wave numbers for $u_{p}(x)=\cos$ $k x+1 / k^{2}$. The results for $k=1$ and $k=10$ are given in Tables 3 and 4, respectively. We see that the schemes are stable against the stronger oscillation in the solution.
Example 5. We extend our method to the case where the source is not of constant strength in this example. We choose a rounded rectangle (Figure $1(\mathrm{c})$ ) with $\rho(t)=(2 / 3)$ $\left[\sin ^{10} t+((2 / 3) \cos t)^{10}\right]^{-0.1}, V=(0.0)^{t}$. The source term is $x^{2}+y+2 / k^{2}$ and the special solution is $u_{p}=\left(x^{2}+y\right) / k^{2}$. The wave number $k$ is taken to be 10 . The results are given 
TABLe 5: Rectangle, $k=10, s=x^{2}+y+2 / k^{2}, u_{p}=\left(x^{2}+y\right) / k^{2}$.

\begin{tabular}{lcccc}
\hline$n$ & B1 & B2 & D1 & D2 \\
\hline 16 & -0.0058494392 & 0.1442299148 & -0.0059015875 & 0.6433536874 \\
32 & 0.2545907391 & 0.0638261490 & 0.2545472731 & 1.3602095013 \\
64 & -0.7497633797 & -2.1618994149 & -0.7497634068 & -2.1815083849 \\
128 & -2.1617145581 & -2.1617145570 & -2.1617145581 & -2.1617145571 \\
256 & -2.1617145570 & -2.1617145570 & -2.1617145570 & -2.1617145570 \\
16 & -0.1044710759 & -0.4635868347 & -0.0835365741 & 1.0986717832 \\
32 & -0.6311738748 & -1.0976862440 & -0.6317799969 & -1.3036324862 \\
64 & 3.5696304769 & 9.1912950343 & 3.5696305220 & 9.2821548762 \\
128 & 9.1908425292 & 9.1908425294 & 9.1908425292 & 9.1908425293 \\
256 & 9.1908425294 & 9.1908425294 & 9.1908425294 & 9.1908425294 \\
\hline
\end{tabular}

in Table 5. This shows the applicability of the proposed method also for a more general source.

\section{Conclusions}

At this place, it is appropriate to draw some conclusive remarks for the proposed $\mathrm{DtN}$ method from our numerical experiments. The source problem is transformed to two boundary value problems for two different domains. In the usual case, only the solution in the exterior to the source is interested. This means that only Problem 2 needs to be solved. The main limitation of the proposed DtN method is the need of a particular solution $u_{p}$ to (1). The form of the source does not matter as long as a particular solution can be found. Once a particular solution is found, it makes no difference if there are other particular solutions to (1). Taking a closer look at equations (22) and (23) for the DtN method, $u_{p}$ appears only at the right side. This means that $u_{p}$ will not affect the solution scheme, neither uniqueness nor stability. Furthermore, in the actual computation, only the values of $u_{p}$ at the collocation points are needed. It follows that even if we do not have an explicit expression for $u_{p}$, the scheme might still work. This includes the case studied in [10]. Finally, we remark that the proposed scheme is very interesting for the corresponding inverse problem which is aimed at reconstructing the unknown source. The attempt of using the same system (22) and (23) to recover the shape and the location of the unknown source can be made in the spirit of [14] where a scattering problem from an impedance obstacle is treated. We also refer to [13] for the recovery of an unknown constant source within a conducting medium modeled by a Laplace equation by a Dirichlet-to-Neumann map.

\section{Data Availability}

No data were used to support this study.

\section{Conflicts of Interest}

The author declares that there are no conflicts of interest.

\section{Acknowledgments}

This work is partially supported by the MOST grant MOST109-2115-M-006-003.

\section{References}

[1] D. Colton and R. Kress, Integral Equation Methods in Scattering Theory, John Wiley and Sons Inc, 1983.

[2] I. Starkgold, Boundary Value Problems of Mathematical Physics, Vol II, the Macmillan Company, New York, 1968.

[3] C. Pozrikids, Boundary Integral and Singularity Methods for Linearized Viscous Flow, Cambridge University Press, Cambridge, 2010.

[4] C. A. Brebbia, The Boundary Element Method for Engineers, Pentech Press, London, 1978.

[5] J. A. Burton, "The solution of Helmholtz equation in exterior domains using integral equation," NPL Report NAC 30, Middlesex, 1973.

[6] R. Kress, Linear Integral Equations, Springer, Berlin, 2nd ed. edition, 1999.

[7] W. C. Chew, Waves and Fields in Inhomogeneous Media, Van Nostrand, New York, 1990.

[8] P. A. Martin, "Acoustic scattering by inhomogeneous obstacles," SIAM Journal on Applied Mathematics, vol. 64, no. 1, pp. 297-308, 2003.

[9] A. Tourin, M. Fink, and A. Derode, "Multiple scattering of sound," Waves in Random Media, vol. 10, no. 4, pp. R31R60, 2000.

[10] R. Rangogni, “The solution of the non-homogeneous Helmholtz equation by means of the boundary element method," Applied Mathematical Modelling, vol. 8, no. 6, pp. 442-444, 1984.

[11] K.-M. Lee, "Transmission scattering problem via a DtN map," Journal of Mathematical Physics, vol. 58, no. 3, article 033502, 2017.

[12] D. Colton and R. Kress, Inverse Acoustic and Electromagnetic Scattering Theory, Springer, Berlin, 2nd ed. edition, 1998.

[13] R. Kress and W. Rundell, "A nonlinear integral equation and an iterative algorithm for an inverse source problem," Journal of Integral Equations and Applications, vol. 27, no. 2, pp. 179198, 2015.

[14] K.-M. Lee, "Inverse scattering problem from an impedance obstacle via two-steps method," Journal of Computational Physics, vol. 274, pp. 182-190, 2014. 